\title{
Long-Term Continuous Antimicrobial Resistance Surveillance Among Nosocomial Gram-Negative Bacilli in China from 2010 to 2018 (CMSS)
}

This article was published in the following Dove Press journal: Infection and Drug Resistance

Qi Wang,' Zhanwei Wang,' Feifei Zhang,' Chunjiang Zhao,' Bin Yang, ${ }^{2}$ Ziyong Sun, ${ }^{3}$ Yaning Mei, ${ }^{4}$ Feng Zhao, ${ }^{5}$ Kang Liao, ${ }^{6}$ Dawen Guo, (iD) ${ }^{7}$ Xiuli Xu, ${ }^{8}$ Hongli Sun, ${ }^{9}$ Zhidong $\mathrm{Hu},{ }^{10}$ Yunzhuo Chu, "I Yi Li, (DD) ${ }^{12}$ Ping ji, ${ }^{13}$ Hui Wang ${ }^{1}$

'Department of Clinical Laboratory, Peking University People's Hospital, Beijing 100044, People's Republic of China; ${ }^{2}$ Department of Clinical Laboratory, The First Affiliated Hospital of Fujian Medical University, Fuzhou 350005, People's Republic of China; ${ }^{3}$ Department of Clinical Laboratory, Tongji Hospital, Tongji Medical College, Huazhong University of Science and Technology, Wuhan 430030, People's Republic of China; ${ }^{4}$ Department of Clinical Laboratory, The First Affiliated Hospital of Nanjing Medical University, Nanjing 210029, People's Republic of China; ${ }^{5}$ Department of Infectious Diseases, Sir Run Shaw Hospital (SRRSH), Affiliated with the Zhejiang University School of Medicine, Hangzhou 310016 , People's Republic of China; ${ }^{6}$ Department of Laboratory Medicine, The First Affiliated Hospital of Sun Yat-Sen University, Guangzhou 510080, People's Republic of China; ${ }^{7}$ Department of Clinical Laboratory, The First Affiliated Hospital of Harbin Medical University, Harbin I5000 I, People's Republic of China; ${ }^{8}$ Department of Clinical Laboratory, Xijing Hospital of Air Force Military Medical University, Xi'an I00I9I, People's Republic of China; ${ }^{9}$ Department of Clinical Laboratory, Peking Union Medical College Hospital, Beijing 100730, People's Republic of China; ${ }^{10}$ Department of Clinical Laboratory, General Hospital of Tianjin Medical University, Tianjin 300052, People's Republic of China; "'Department of Clinical Laboratory, The First Affiliated Hospital of China Medical University, Shenyang I I000 I, People's Republic of China; ${ }^{12}$ Department of Clinical Laboratory, Henan Provincial People's Hospital, Zhengzhou 450003, People's Republic of China; ${ }^{13}$ Department of Clinical Laboratory, The First Affiliated Hospital of Xinjiang Medical University, Urumqi 830054, People's Republic of China

Correspondence: Hui Wang

Department of Clinical Laboratory, Peking University People's Hospital, Beijing 100044 , People's Republic of China

$\mathrm{Tel} / \mathrm{Fax}+86-10-88326300$

Email whuibj@I63.com
Purpose: The Chinese Meropenem Surveillance Study (CMSS) was conducted every 2 years from 2010 to 2018 to monitor the antimicrobial activity of commonly used antimicrobial agents against nosocomial gram-negative bacilli in China.

Methods: From 2010 to 2018, 6,537 gram-negative bacilli were collected from 14 teaching hospitals. The minimum inhibitory concentrations (MICs) of meropenem and other antimicrobial agents were determined using the agar dilution and broth microdilution methods.

Results: Continuous surveillance indicated that, except for Klebsiella pneumoniae, the susceptibility of Enterobacterales to carbapenems was relatively stable over time. Carbapenems had the highest activity against the tested isolates, with $\mathrm{MIC}_{90}$ values (MIC for $90 \%$ of organisms) ranging from $0.032 \mathrm{mg} / \mathrm{L}$ to $8 \mathrm{mg} / \mathrm{L}$. More than $90 \%$ of bacteria were susceptible to either meropenem or imipenem; more than $80 \%$ were susceptible to ertapenem. The prevalence of extended-spectrum beta-lactamase (ESBL)-producing E. coli, $K$. pneumoniae, and P. mirabilis each year was 50.4-64.3\%, 18-41.2\%, and $1.9-33.8 \%$, respectively. The prevalence of carbapenem-resistant $K$. pneumoniae (CRKP) and carbapenem-resistant Acinetobacter baumannii (CRAB) continued to increase significantly over time, from $7.6 \%$ to $21.2 \%$ and $64.6 \%$ to $69.3 \%$, respectively. The prevalence of CRKP was higher from urinary tract infections $(25.4 \%)$ than from bloodstream infections $(14.2 \%)$, intra-abdominal infections (14.5\%), and respiratory infections (14.4\%). In total, 129 CRKP isolates were evaluated by PCR; of these, $92(71.3 \%)$ carried the $b l a_{\mathrm{KPC}-2}$ gene. Colistin maintained very high in vitro antimicrobial activity against $P$. aeruginosa and A. baumannii (more than $95 \%$ of isolates exhibited susceptibility at all timepoints).

Conclusion: The results indicate an increase in $K$. pneumoniae resistance to carbapenems over time, mainly owing to KPC-type carbapenemase production. A. baumannii was severely resistant to carbapenems in China. Ongoing MIC-based resistance surveillance, like CMSS, provides additional data for clinical anti-infective treatment.

Keywords: CMSS, gram-negative bacilli, antimicrobial susceptibility surveillance, carbapenem-resistant

\section{Introduction}

In recent years, the proliferation of various multidrug-resistant gram-negative bacteria, such as extended-spectrum beta-lactamase (ESBL)-producing Enterobacterales, carbapenem-resistant Enterobacterales (CRE), carbapenem-resistant Acinetobacter baumannii (CRAB), carbapenem-resistant Pseudomonas aeruginosa, and other carbapenemresistant gram-negative bacteria, have introduced new challenges to clinical antiinfectious disease treatment and hospital infection control. ${ }^{1-5}$ In 2019 , in the latest list 
of antibiotic-resistance threats released by the United States $\mathrm{CDC}$, the number of drug-resistant bacteria identified in the report had increased from the previous version; ${ }^{6}$ this reality makes the clinical challenge of combatting multidrug-resistant bacteria even more complex. However, the prevalence of these multidrug-resistant bacteria in different countries and regions is not uniform. Additionally, the prevalence of multidrugresistant bacteria changes over time. Factors affecting the prevalence of drug-resistant bacteria include region, population, clinical infection type, and local prescription behavior. ${ }^{7,8}$ Therefore, timely and effective antimicrobial susceptibility surveillance is essential for epidemiology, infection control, and empirical antimicrobial agent prescriptions.

The Meropenem Yearly Susceptibility Test Information Collection (MYSTIC) programme ${ }^{9-12}$ was initiated in 1997 with the primary purpose of monitoring changes in the susceptibility of specific bacteria to clinically relevant antibacterial agents such as meropenem. At present, most antimicrobial susceptibility surveillance projects in China are based on historical data review, and there are few projects engaged in the prospective collection of isolates. The Chinese Meropenem Susceptibility Surveillance (CMSS) project was initiated in $2003 .{ }^{13}$ Under this project, surveillance of bacterial infections has been performed every 2 years, mainly regarding the susceptibility of specific Enterobacterales and non-fermentative bacteria to antimicrobial agents commonly used in China. In this article, we report and summarize the CMSS data from 2010 to 2018. We expect that our results will contribute to both empiric therapy and infection control in the antiinfection field.

\section{Materials and Methods}

\section{Bacterial Isolates Collection}

Nine Enterobacterales species and three non-fermentative bacterial species were collected, including Escherichia coli, Klebsiella pneumoniae, Enterobacter cloacae, Klebsiella aerogenes, Citrobacter freundii, Serratia marcescens, Morgan morganii, Proteus mirabilis, Proteus vulgaris, Acinetobacter baumannii, Pseudomonas aeruginosa, and Burkholderia cepacia. All isolates were part of the routine hospital laboratory procedure.

From 2010 to 2018, CMSS surveillance was conducted every 2 years, in a total of five collection rounds. The surveillance years were 2010, 2012, 2014, 2016, and 2018. Thirteen teaching hospitals from 11 central cities (Beijing, Tianjin, Shenyang, Shanghai, Hangzhou, Zhengzhou,
Wuhan, Nanjing, Guangzhou, Fuzhou, and Urumqi) throughout China participated in the CMSS program. From March to August each year, 100 non-repeat clinical isolates of gramnegative bacilli were collected in the hospitals. Isolates were identified at the local laboratory and confirmed at the central laboratory (Department of Clinical Laboratory, Peking University People's Hospital, Beijing, China) using colonial morphology, routine biochemical tests, and Vitek system identification (bioMérieux, Hazelwood, MO, USA), as required. All isolates were stored at $-80{ }^{\circ} \mathrm{C}$ until the MICs were measured.

\section{Antimicrobial Susceptibility Testing}

The MICs of 15 antimicrobial agents were determined for each isolate using the agar dilution method or the broth microdilution method at the central laboratory according to the Clinical and Laboratory Standards Institute (CLSI) guidelines. ${ }^{14}$ For colistin and tigecycline, the broth microdilution methods were used to determine MICs for all isolates, while agar dilution methods were used to determine MICs for other antibacterial agents.

Mueller-Hinton agar (BBL Microbiology Systems, Cockeysville, MD, USA) was freshly prepared for susceptibility testing. The antimicrobial agents tested were meropenem (Sumitomo Pharmaceuticals Co., Osaka, Japan), imipenem (Sigma Chemical Co., St Louis, MO, USA), ertapenem (Sigma), ceftazidime (Sigma), cefotaxime (Sigma), ceftriaxone (Sigma), cefepime (Sigma), piperacillin/tazobactam (TZP; Wyeth Pharmaceuticals, Collegeville, PA, USA), cefoperazone/sulbactam (CSL, 2:1; Sigma), clavulanic acid (Sigma), cefoxitin (Sigma), amikacin (Sigma), ciprofloxacin (Bayer AG, Leverkusen, Germany), levofloxacin (Bayer AG), tigecycline (MedChem Express, Monmouth Junction, NJ, USA), and colistin (Sigma). The procedures for each set of tests were validated by determining the MICs for quality control isolates (Escherichia coli ATCC 25922, Klebsiella pneumonia ATCC 700603 and Pseudomonas aeruginosa ATCC 27853) as recommended by the CLSI standards. ${ }^{14}$ The results were interpreted according to the most recent CLSI M100-S29 breakpoints. ${ }^{14}$ The cefoperazonesulbactam MIC breakpoint used the breakpoint of cefoperazone for Enterobacterales in the CLSI M100-S29. ${ }^{14}$ Tigecycline MICs interpretation refers to the breakpoint of the US FDA (www.fda.gov/drugs/developmentresources/tigecycline-injection-products). Colistin MICs interpretation refers to the breakpoint of the European 
Committee on Antimicrobial Susceptibility Testing (EUCAST). ${ }^{15}$

The CLSI extended-spectrum beta-lactamase (ESBL)screening criterion (MIC $\geq 2 \mathrm{mg} / \mathrm{L}$ for either ceftazidime or cefotaxime) was applied to all the E. coli, K. pneumoniae, and P. mirabilis isolates. ESBL production was confirmed using two drug pairs, cefotaxime alone or cefotaxime plus clavulanic acid and ceftazidime alone or ceftazidime plus clavulanic acid. An isolate was considered ESBLproducing if the addition of clavulanic acid reduced the MIC of either of the beta-lactam agents by three-fold or more. Escherichia coli ATCC 25922 and Klebsiella pneumoniae ATCC 700603 were used as controls for the confirmatory ESBL test.

\section{Carbapenemase Gene Detection}

The six primary carbapenemase genes $\left(b l a_{\mathrm{KPC}}, b l a_{\mathrm{NDM}}\right.$, bla $_{\mathrm{IMP}}$, bla $_{\mathrm{VIM}}$, bla $_{\mathrm{SIM}}$, and bla $\left.a_{\mathrm{OXA}-48}\right)$ of 233 CRE isolates were amplified using PCR as previously described. ${ }^{16,17}$ The PCR products were purified using a Universal DNA Purification Kit (Tiangen Biotech, Beijing, China) and sequenced by Sanger sequencing on an ABI PRISM 3730XL system (Applied Biosystems, Foster City, CA, USA). The sequences were aligned using the NCBI BLAST tool to determine the specific carbapenemase genotype.

\section{Data Analysis and Statistical Analysis}

Case reports, including the patient's clinical diagnosis, the date of collection specimen, and the type of infection, were collected along with the strains. All the antimicrobial susceptibility test data were analyzed by WHONET 5.6.

\section{Ethical Statement}

This study was approved by the Ethics Review Committee (ERC) of Peking University People's Hospital. Informed consent was not needed due to that the medical records and patient information were anonymously reviewed and collected.

\section{Results}

\section{Distribution of Isolates}

From 2010 to 2018, in total, 6,537 gram-negative bacilli were collected. The distribution of organisms was: Escherichia coli (1022/6537, 15.6\%), Klebsiella pneumoniae (983/6537, 15\%), Acinetobacter baumannii (926/ 6537, 14.2\%), Pseudomonas aeruginosa (922/6537,
14.1\%), Enterobacter cloacae (829/6537, 12.7\%), Citrobacter freundii (398/6537, 6.1\%), Proteus mirabilis (333/6537, 5.1\%), Serratia marcescens (315/6537, 4.8\%), Klebsiella aerogenes (303/6537, 4.6\%), Morgan morganii (214/6537, 3.3\%), Burkholderia cepacia (209/6537, $3.2 \%)$, and Proteus vulgaris $(83 / 6537,1.3 \%)$. The majority of the isolates were recovered from blood culture specimens $(2589 / 6537,39.6 \%)$, followed by urine $(836 / 6537$, $12.8 \%)$, sputum $(803 / 6537,12.3 \%)$, drainage $(353 / 6537$, $5.4 \%)$, secretion $(305 / 6537,4.7 \%)$, pus $(261 / 6537,4 \%)$, abdominal fluid $(252 / 6537,3.9 \%)$, bile $(213 / 6537,3.3 \%)$, wound (146/6537, 2.2\%), pleural fluid (121/6537, 1.9\%), cerebrospinal fluid $(109 / 6537,1.7 \%)$, catheter $(92 / 6537$, $1.4 \%)$, broncho-alveolar lavage $(66 / 6537,1 \%)$, and other specimens $(391 / 6537,6 \%)$.

\section{Antimicrobial Activity Against Major Organisms from 2010 to 2018}

The antimicrobial activity against major organisms from 2010 to 2018 is described in Table 1. During this period, the susceptibility of $E$. coli to carbapenems remained between $91.9 \%$ and $100 \%$. The susceptibility of E. coli to ceftriaxone and cefotaxime was between $29.8 \%$ and $38.9 \%$, but the susceptibility to ceftazidime remained between $63.2 \%$ and $65.6 \%$. More than $95 \%$ of E. coli were susceptible to tigecycline and colistin in each monitoring year. There was no significant change in the $\mathrm{MIC}_{90}$ data of the 15 antimicrobials against E. coli.

The susceptibility of $K$. pneumoniae to meropenem, imipenem, and ertapenem decreased from 93.5\%, 93.5\%, and $91.8 \%$ in 2010 to $79.7 \%, 80.2 \%$, and 78.4 in 2018 , respectively. In 2010 , the $\mathrm{MIC}_{90}$ data of $K$. pneumoniae against meropenem, imipenem, and ertapenem were $0.064 \mathrm{mg} / \mathrm{L}, 0.5 \mathrm{mg} / \mathrm{L}$, and $0.5 \mathrm{mg} / \mathrm{L}$, respectively. In 2018 , the $\mathrm{MIC}_{90}$ data of $K$. pneumoniae against meropenem, imipenem, and ertapenem increased to $64 \mathrm{mg} / \mathrm{L}$, $16 \mathrm{mg} / \mathrm{L}$, and $256 \mathrm{mg} / \mathrm{L}$, respectively. K. pneumoniae susceptibility to several antimicrobial agents was significantly reduced over time, including susceptibility to cefoxitin (from $81.1 \%$ in 2010 to $67.6 \%$ in 2018), piperacillintazobactam (from $87.1 \%$ in 2010 to $73.4 \%$ in 2018 ), amikacin (from $90 \%$ in 2010 to $85.1 \%$ in 2018). K. pneumoniae susceptibility to colistin remained between $98.2 \%$ and $99.5 \%$. In all of the years except for 2010 , the susceptibility of $K$. pneumoniae to tigecycline remained above $90 \%$. 


\begin{tabular}{|c|c|c|c|c|c|}
\hline \multirow[b]{3}{*}{$\frac{\infty}{2}$} & $\frac{8}{\Sigma}$ & \multirow[b]{3}{*}{$\underset{\widetilde{N}}{\stackrel{N}{I}}$} & 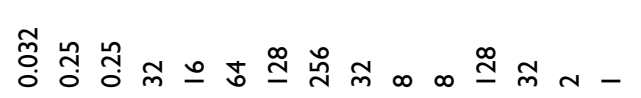 & \multirow[b]{3}{*}{$\underset{\mathbb{Z}}{\mathbb{Z}}$} & 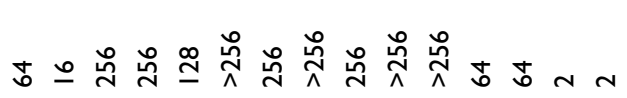 \\
\hline & ஃ̊ & & 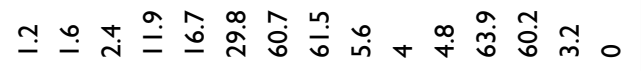 & & 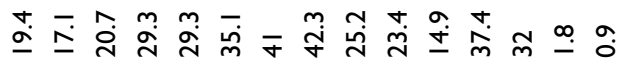 \\
\hline & ஸे & & œ & & 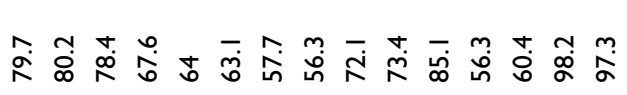 \\
\hline & $\frac{\stackrel{0}{\Sigma}}{\Sigma}$ & \multirow[b]{3}{*}{$\stackrel{\infty}{\Perp}$} & 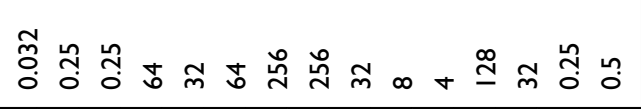 & \multirow[b]{3}{*}{ 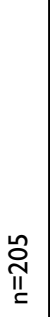 } & 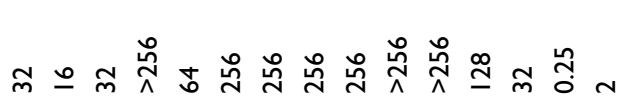 \\
\hline & ஃ̊ & & 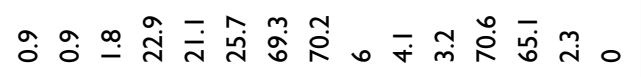 & & 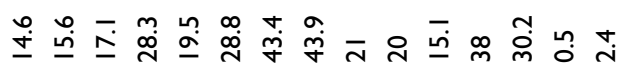 \\
\hline$\stackrel{4}{\circ}$ & iे & & 我 & & 芦 ̃ \\
\hline & $\frac{8}{\Sigma}$ & \multirow[b]{3}{*}{$\frac{\infty}{\stackrel{\infty}{I}}$} & 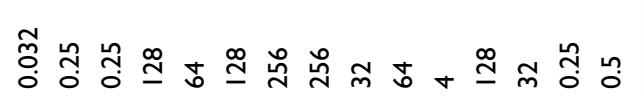 & \multirow[b]{3}{*}{$\frac{\alpha}{\frac{\alpha}{11}}$} & 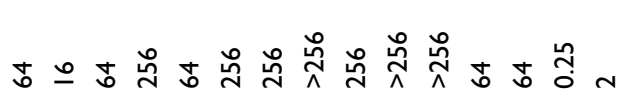 \\
\hline & ஃ̊ & & 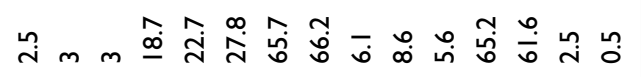 & & 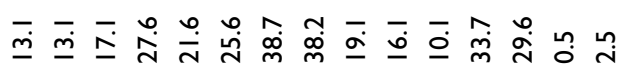 \\
\hline$\frac{4}{2}$ & ஸे & & 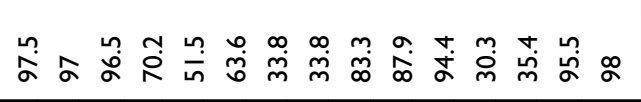 & & 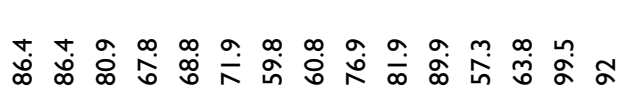 \\
\hline & $\frac{\stackrel{0}{\Sigma}}{\Sigma}$ & \multirow[b]{3}{*}{$\underset{\infty}{\stackrel{\infty}{I}}$} & 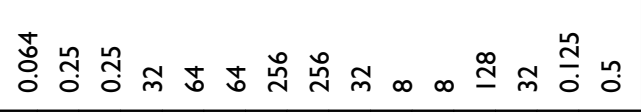 & \multirow[b]{3}{*}{$\frac{\widehat{\omega}}{11}$} & 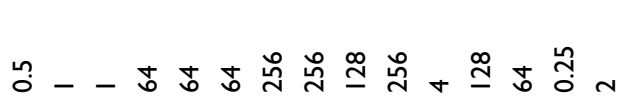 \\
\hline & ஓ̊ & & ○ ○ ڤ & & 冓 虽 \\
\hline$\frac{4}{2}$ & iे & & 으응 芦 & & 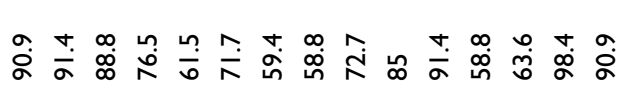 \\
\hline & $\frac{0^{\circ}}{\Sigma}$ & \multirow[b]{3}{*}{$\frac{\sqrt{n}-1}{n}$} & 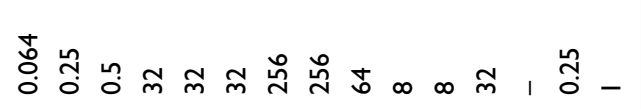 & \multirow[b]{3}{*}{$\stackrel{\pi}{I}$} & 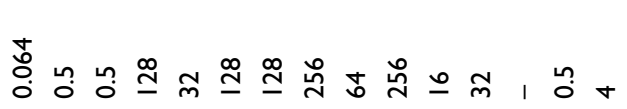 \\
\hline & ஓ̊ & & 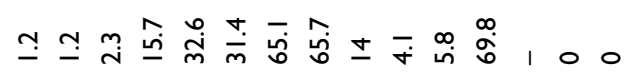 & & 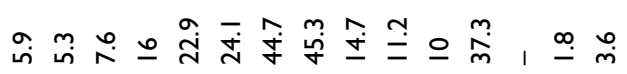 \\
\hline$\frac{\text { 음 }}{2}$ & iे & & 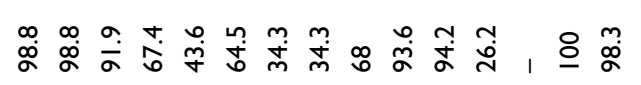 & & 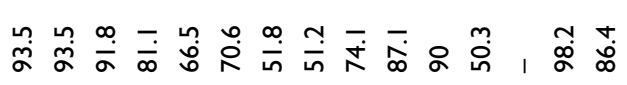 \\
\hline \multicolumn{2}{|c|}{ 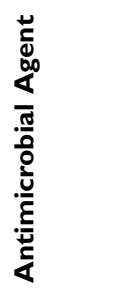 } & \multicolumn{2}{|r|}{ 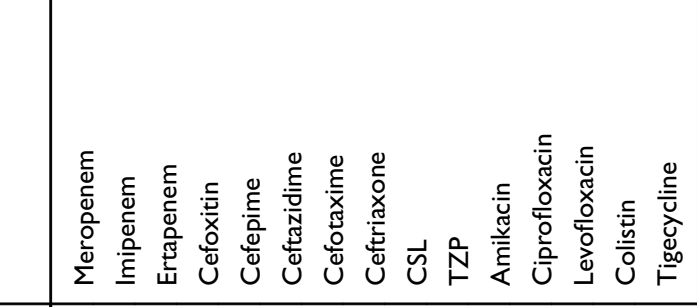 } & \multicolumn{2}{|r|}{ 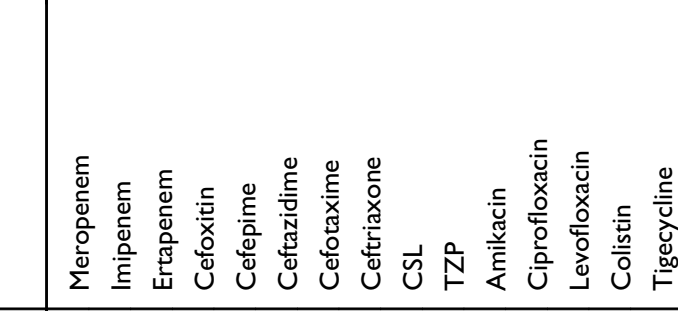 } \\
\hline 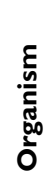 & & $\overline{\text { वे }}$ & & 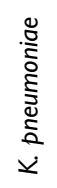 & \\
\hline
\end{tabular}




\begin{tabular}{|c|c|c|c|c|c|}
\hline \multirow[b]{3}{*}{$\stackrel{\stackrel{m}{m}}{\frac{m}{11}}$} & 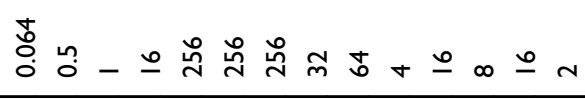 & & 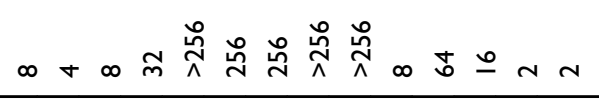 & \multirow[b]{3}{*}{$\underset{\text { II }}{\text { II }}$} & 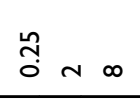 \\
\hline & 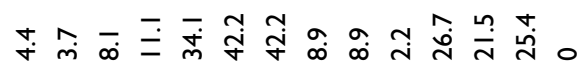 & & 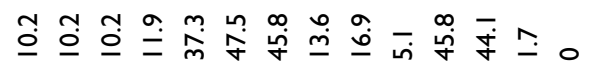 & & $\underset{\infty}{\sim} \underset{\infty}{\sim} \stackrel{\simeq}{\simeq}$ \\
\hline & 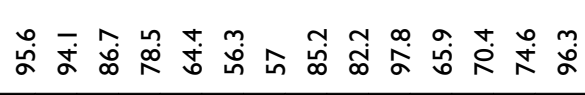 & 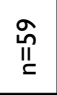 & 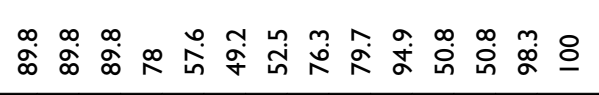 & & 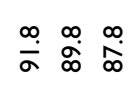 \\
\hline \multirow[b]{3}{*}{$\frac{\dddot{m}}{11}$} & 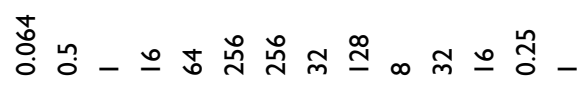 & & 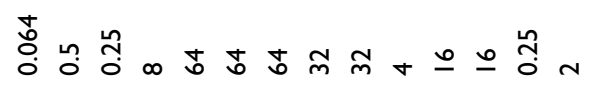 & \multirow[b]{3}{*}{ 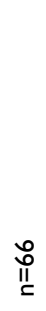 } & $\begin{array}{l}\stackrel{t}{0} \\
\stackrel{0}{0} \\
0\end{array}$ \\
\hline & 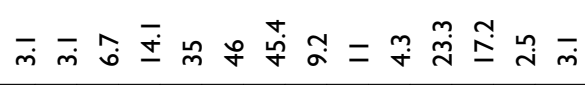 & & 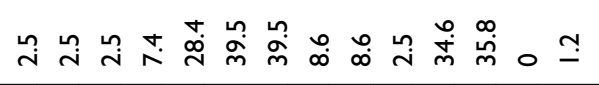 & & $\stackrel{\leftrightarrow}{+} \stackrel{\varphi}{+} \bar{m}$ \\
\hline & 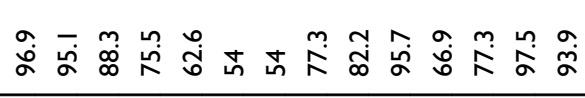 & 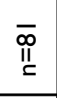 & 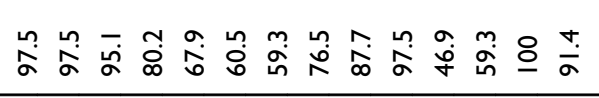 & & 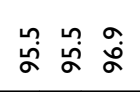 \\
\hline \multirow[b]{3}{*}{$\frac{\bar{\sigma}}{\underline{11}}$} & 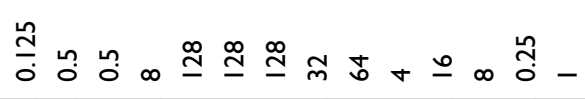 & & 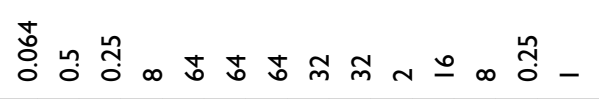 & \multirow[b]{3}{*}{$\stackrel{0}{i}$} & $\stackrel{n}{\stackrel{0}{0}} \sim \infty$ \\
\hline & 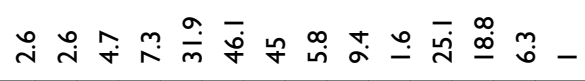 & & 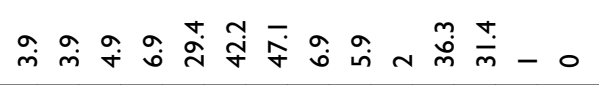 & & 으으 $\overline{0}$ \\
\hline & 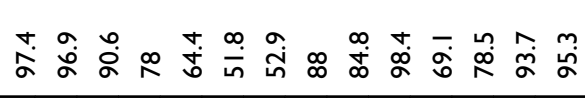 & $\frac{\delta}{\Perp}$ & 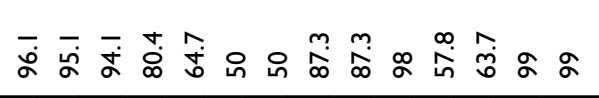 & & 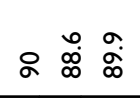 \\
\hline \multirow[b]{3}{*}{$\frac{\hat{0}}{11}$} & 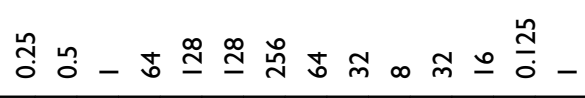 & & 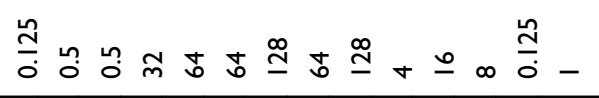 & \multirow[b]{3}{*}{ 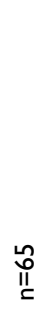 } & 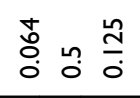 \\
\hline & 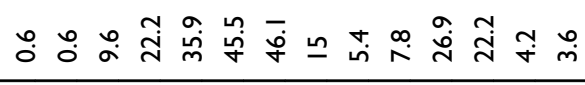 & & 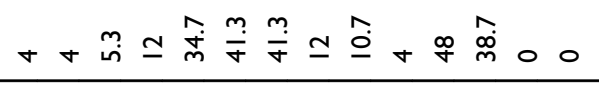 & & 느 는 눔 \\
\hline & 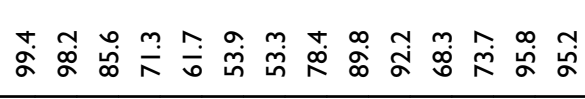 & 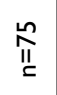 & ஃ。 & & 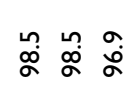 \\
\hline \multirow[b]{3}{*}{$\frac{m}{I !}$} & 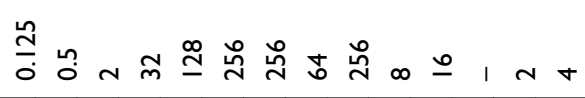 & & 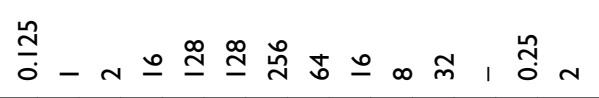 & \multirow[b]{3}{*}{ 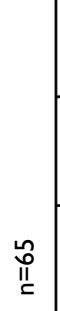 } & $\stackrel{+}{\stackrel{0}{0}}-\frac{\stackrel{L}{0}}{0}$ \\
\hline & 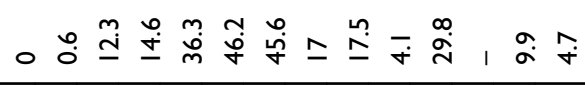 & & 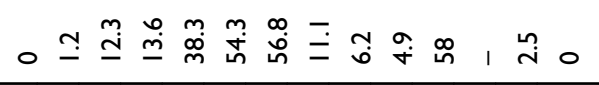 & & $00 \stackrel{\text { ำ }}{-}$ \\
\hline & 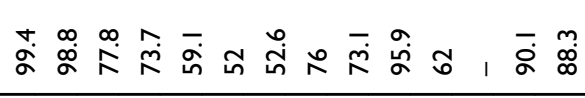 & 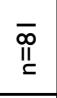 & 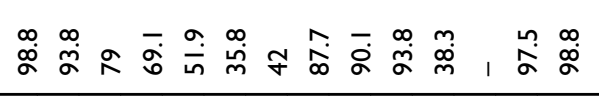 & & 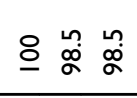 \\
\hline & 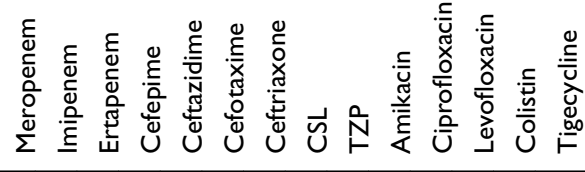 & & 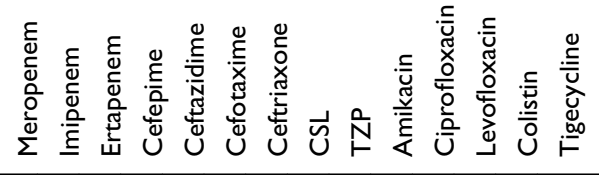 & & 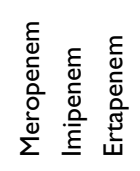 \\
\hline 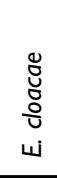 & & $\dot{v}$ & & 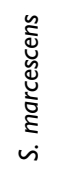 & \\
\hline
\end{tabular}




\begin{tabular}{|c|c|c|c|c|c|c|}
\hline \multirow[b]{3}{*}{$\frac{\infty}{2}$} & $\frac{\stackrel{8}{\Sigma}}{\Sigma}$ & $\simeq \simeq \stackrel{\infty}{\simeq} \stackrel{\infty}{\simeq} \tilde{m} \stackrel{\infty}{\simeq} \sim+\infty-$ & \multirow[b]{3}{*}{$\stackrel{a}{\stackrel{a}{I}}$} & 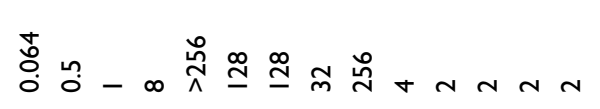 & \multirow[b]{3}{*}{$\stackrel{+}{\stackrel{L}{I}}$} & 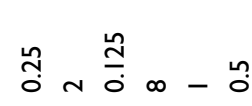 \\
\hline & ळ̊ & 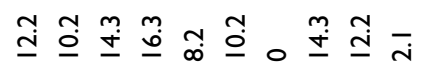 & & 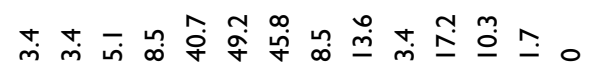 & & $0 \stackrel{+}{\sim} 0 \stackrel{a}{-} 0 \stackrel{a}{.}$ \\
\hline & ڤे & 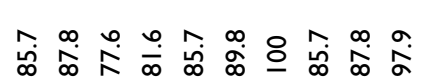 & & 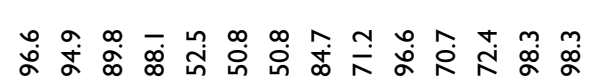 & & 으 땡으 영으 영 \\
\hline \multirow[b]{3}{*}{ 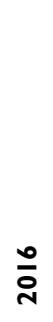 } & $\frac{\stackrel{0}{0}}{\Sigma}$ & 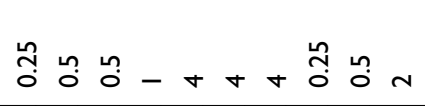 & \multirow[b]{3}{*}{ 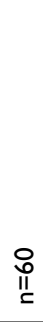 } & 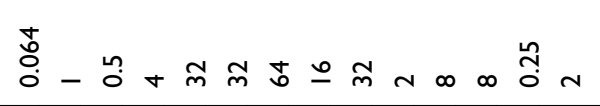 & \multirow[b]{3}{*}{$\stackrel{\infty}{\|}$} & 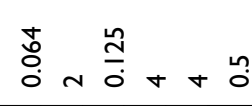 \\
\hline & œ̊ & 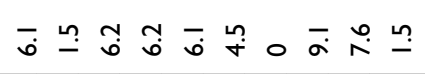 & & 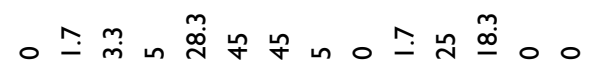 & & $\circ \stackrel{0}{i} \circ 0 \stackrel{i}{m}$ \\
\hline & i̊ & సूં & & & & 응 \\
\hline \multirow[b]{3}{*}{$\frac{4}{\pi}$} & $\frac{\stackrel{0}{\Sigma}}{\Sigma}$ & 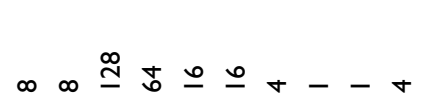 & \multirow[b]{3}{*}{ 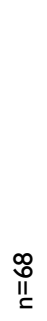 } & 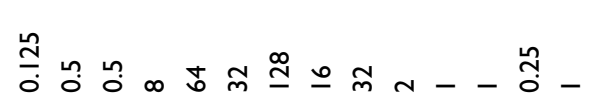 & \multirow[b]{3}{*}{$\underset{11}{0}$} & 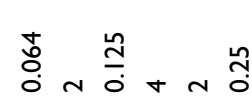 \\
\hline & ஓ̊ & 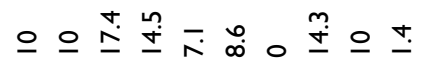 & & 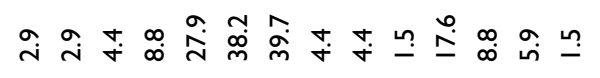 & & 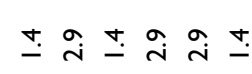 \\
\hline & ڤ̊ & 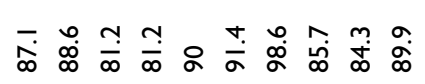 & & 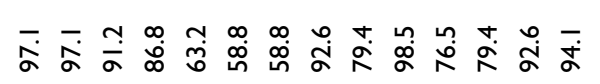 & & 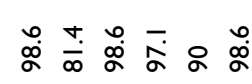 \\
\hline \multirow[b]{3}{*}{$\frac{4}{4}$} & $\frac{0}{\Sigma}$ & $\infty \sigma \dot{\Xi} \stackrel{\infty}{\simeq} \varrho \sigma+--N$ & \multirow[b]{3}{*}{$\stackrel{0}{0}$} & 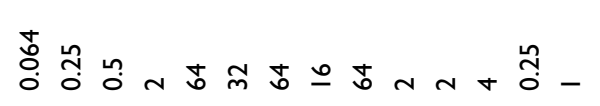 & \multirow[b]{3}{*}{$\stackrel{\circ}{i}$} & 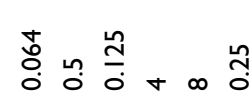 \\
\hline & ஓ̊ & 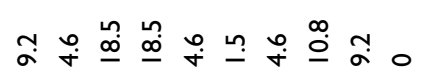 & & 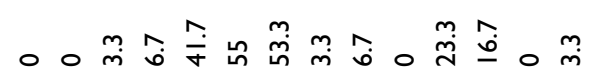 & & 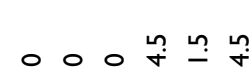 \\
\hline & ڤ̊ำ & 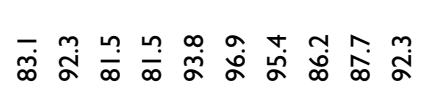 & & 으으 & & 으으으 占 \\
\hline \multirow[b]{3}{*}{$\frac{0}{2}$} & $\frac{8}{\Sigma}$ & $\infty \sim \tilde{m} \tilde{m} \mathscr{\sigma}+-\cdots$ & \multirow{4}{*}{\multicolumn{2}{|c|}{ 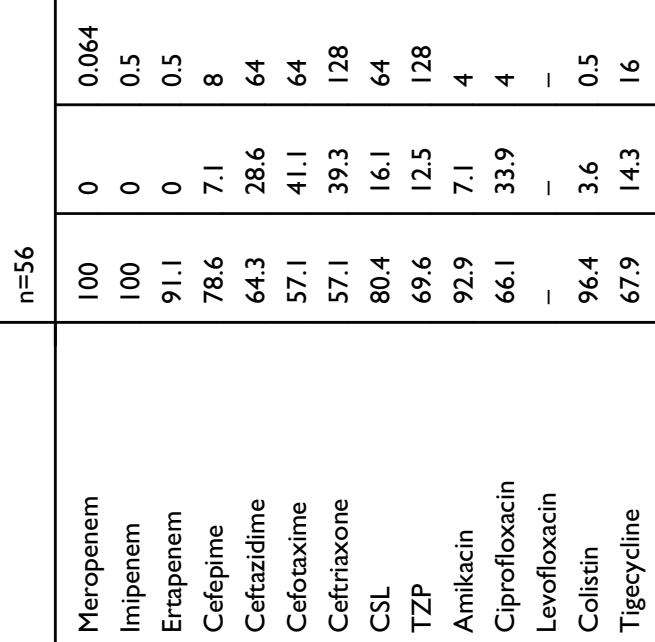 }} & \multirow[b]{3}{*}{ 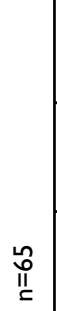 } & $\stackrel{+}{\circ} \stackrel{\circ}{\circ}+\stackrel{0}{0}+\infty \stackrel{n}{0}$ \\
\hline & œ̊ & ๙ั & & & & $\circ$ 이일 \\
\hline & ڤ̊ํ & 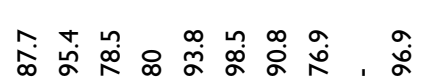 & & & & 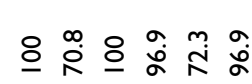 \\
\hline \multicolumn{2}{|l|}{ 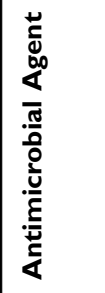 } & 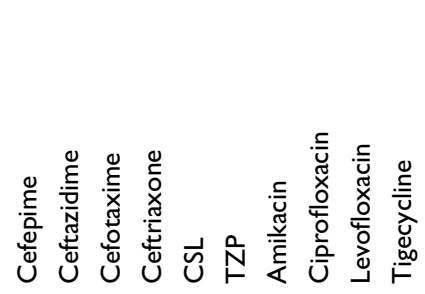 & & & & 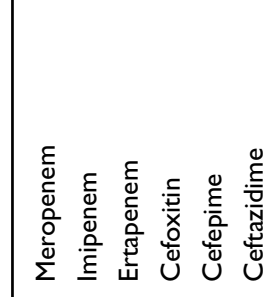 \\
\hline 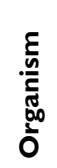 & & & 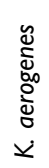 & & 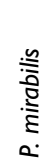 & \\
\hline
\end{tabular}




\begin{tabular}{|c|c|c|c|c|}
\hline$\tilde{m} \tilde{m}+\stackrel{\llcorner}{0} \infty \approx \tilde{m} \infty$ & \multirow[b]{3}{*}{$\frac{\tilde{n}}{11}$} & \multirow{2}{*}{ 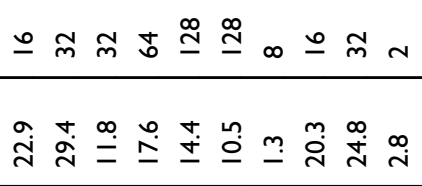 } & \multirow[b]{3}{*}{$\frac{m}{11}$} & 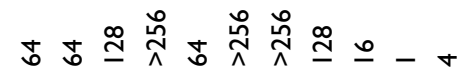 \\
\hline 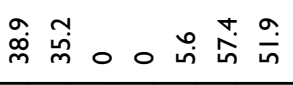 & & & & 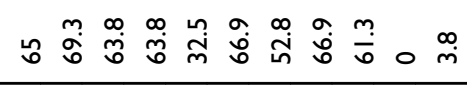 \\
\hline$\overline{\bar{\sigma}}$ ๓ & & バ ஃ & & 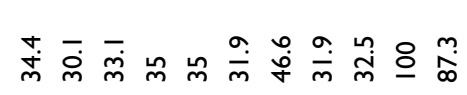 \\
\hline$\underline{\simeq} \infty++\overleftarrow{t} \infty$ & & 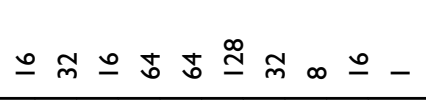 & & 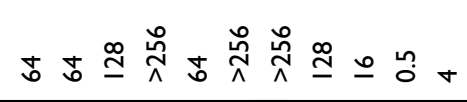 \\
\hline 䃾 & & 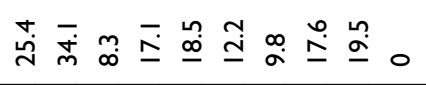 & & 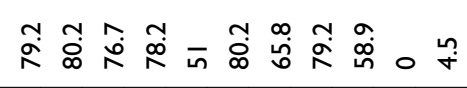 \\
\hline 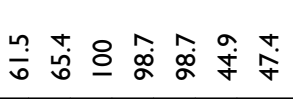 & 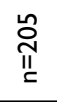 & 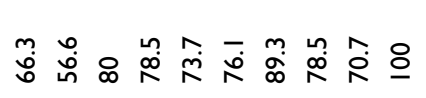 & $\underset{\text { II }}{\check{I}}$ & 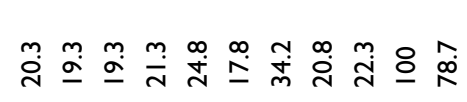 \\
\hline$\infty \infty+-\sim \stackrel{\infty}{=}$ & & 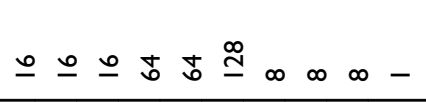 & & 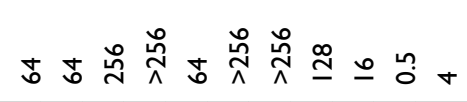 \\
\hline 穴 & & 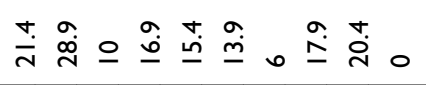 & & 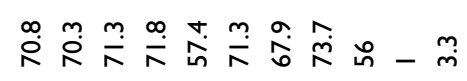 \\
\hline 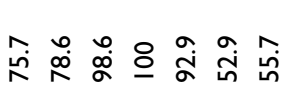 & $\overline{\stackrel{i}{\pi}}$ & 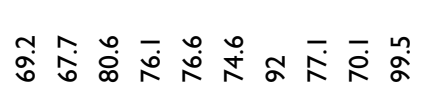 & 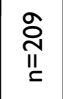 & 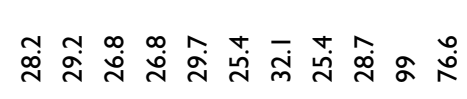 \\
\hline$\underline{0}+-\sigma \stackrel{m}{\infty}$ & & 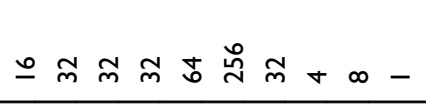 & & 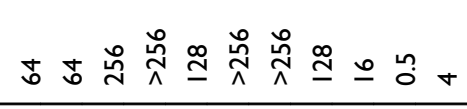 \\
\hline 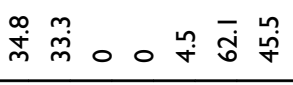 & & 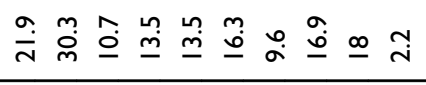 & & 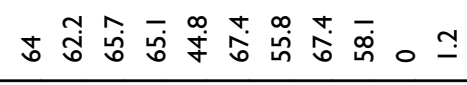 \\
\hline 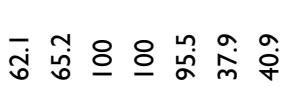 & $\stackrel{\infty}{\frac{\infty}{11}}$ & 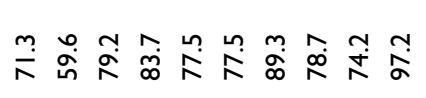 & $\frac{\sqrt{11}}{\frac{1}{N}}$ & 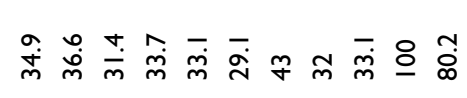 \\
\hline$\infty \infty+-\sigma \tilde{m}$ & & 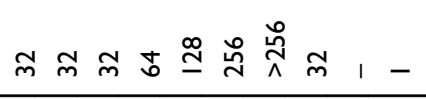 & & 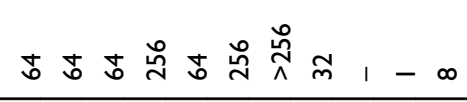 \\
\hline 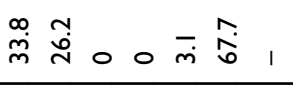 & & 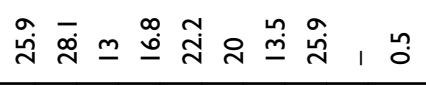 & & 芦 \\
\hline 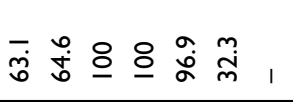 & 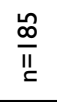 & 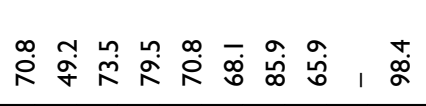 & $\frac{\infty}{11}$ & 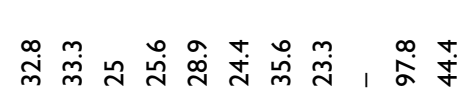 \\
\hline 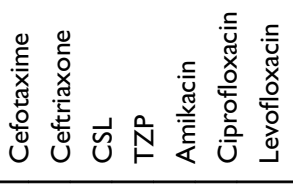 & & 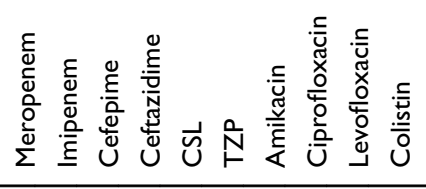 & & 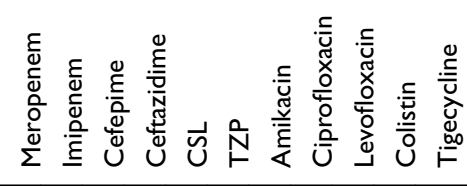 \\
\hline & 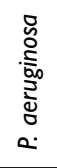 & & 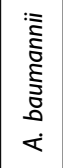 & \\
\hline
\end{tabular}


The susceptibility of E. cloacae to meropenem and imipenem decreased each year, from $98.8-99.4 \%$ in 2010 to $94.1-95.6 \%$ in 2018 . In each year, the monitoring results showed that the proportion of E. cloacae resistant to colistin was between $2.5-25.4 \%$.

The susceptibility of $C$. freundii to meropenem and imipenem decreased from $98.8 \%$ and $93.8 \%$ in 2010 , respectively, to $89.8 \%$ to both drugs in 2018 . Simultaneously, the $\mathrm{MIC}_{90}$ increased to $8 \mathrm{mg} / \mathrm{L}$ and $4 \mathrm{mg} / \mathrm{L}$ for meropenem and imipenem, respectively. The susceptibility rate of $C$. freundii to cefoperazone-sulbactam and piperacillin-tazobactam was more than $75 \%$ in all the years tested; the susceptibility to amikacin, colistin, and tigecycline was over $90 \%$.

From 2010 to 2018, the susceptibility of $P$. aeruginosa to meropenem increased from $70.8 \%$ to $73.2 \%$, and the susceptibility to imipenem increased from $49.2 \%$ to $66 \%$. Simultaneously, the $P$. aeruginos $a$ isolates exhibited increased susceptibility to cefepime, ceftazidime, cefoperazone-sulbactam, piperacillintazobactam, and amikacin. The susceptibility of $P$. aeruginosa to ciprofloxacin and levofloxacin was reduced over time.

A. baumannii susceptibility to meropenem and imipenem was less than $40 \%$ in all of the study years. The $\mathrm{MIC}_{90}$ of A. baumannii for piperacillin-tazobactam, amikacin, and ceftazidime was higher than $128 \mathrm{mg} / \mathrm{L}$, and the $\mathrm{MIC}_{90}$ for cefoperazone-sulbactam was between $64-128 \mathrm{mg} / \mathrm{L}$. A. baumannii resistance to colistin was less than $3 \%$.

\section{Multi-Drug-Resistant Bacteria from 2010 to 2018}

Information on the major resistant gram-negative bacilli in each surveillance year is listed in Table 2. Among carbapenem-resistant Enterobacterales, the incidence of carbapenem-resistant $K$. pneumoniae was increased each year, from $7.6 \%$ in 2010 to $21.2 \%$ in 2018 . Among the other Enterobacterales isolates, higher carbapenem resistance rates were observed in $C$. freundii $(2.5-12.3 \%)$, S. marcescens (1.5-12.2\%), and E. cloacae (4.7-12.1\%). The incidence of carbapenem-resistant E. coli varied between $0.5 \%$ and $3.5 \%$. From 2010 to 2016, the incidence of carbapenem-resistant $A$. baumannii increased significantly from $64.4 \%$ to $80.2 \%$ (with an incidence of $69.3 \%$ in 2018 ). The incidence of ESBL-producing $E$. coli fluctuated between $50.4 \%$ and $64.3 \%$. Using the CLSI phenotypic confirmation method, we found that the proportion of ESBL produced by $K$. pneumoniae decreased from $41.2 \%$ in 2010 to $18 \%$ in 2018. The prevalence of multidrug-resistant bacteria in the different infection types is shown in Table 3 . We analyzed specimens of the four major infection sources separately, including bloodstream infections (BSIs), intra-abdominal infections (IAIs), respiratory infections (RIs), and urinary tract infections (UTIs). The prevalence of CRKP (25.4\%, 18/71) in UTIs was higher than that of CRKP in BSIs $(27.7 \%, 156 / 564)$, IAIs $(26.7 \%, 35 / 131)$, and RIs $(29.8 \%$, $31 / 104)$. The prevalence of CRAB $(56.4 \%, 22 / 39)$ in UTIs was lower than that of CRAB in BSIs $(73.2 \%, 298 / 407)$, IAIs (72.4\%, 84/116), and RIs (72.9\%, 145/199). ESBLproducing E. coli, K. pneumoniae, and P. mirabilis from the urinary tract were also more prevalent than the other three types of infection. The proportion of carbapenem-resistant Serratia marcescens $(12.3 \%, 10 / 81)$ in BSIs was higher than that in IAIs $(6.9 \%, 2 / 29)$, RIs $(5.7 \%, 7 / 122)$, and UTI $(0 \%, 0 / 38)$.

Table 2 Prevalence by Year of Multidrug-Resistant Gram-Negative Isolates Isolates

\begin{tabular}{|c|c|c|c|c|c|}
\hline \multirow[t]{2}{*}{ Organism } & \multicolumn{5}{|c|}{ \% (No. of Isolates) } \\
\hline & 2010 & 2012 & 2014 & 2016 & 2018 \\
\hline CR-C. freundii & $12.3(10 / 81)$ & $5.3(4 / 75)$ & $4.9(5 / 102)$ & $2.5(2 / 8 I)$ & $10.2(6 / 59)$ \\
\hline CR-K. aerogenes & $0(0 / 56)$ & $3.3(2 / 60)$ & $4.4(3 / 68)$ & $3.3(2 / 60)$ & $5.1(3 / 59)$ \\
\hline CR-E. cloacae & I2.I (2I/I73) & $9.6(16 / 167)$ & $4.7(9 / 191)$ & $6.7(11 / 163)$ & $8.1(11 / 135)$ \\
\hline CR-E. coli & $2.3(4 / 172)$ & $0.5(\mathrm{I} / 182)$ & $3.5(7 / 198)$ & $1.8(4 / 2 \mid 8)$ & $2.4(6 / 252)$ \\
\hline CR-K. pneumoniae & $7.6(13 / 170)$ & $9.6(18 / 187)$ & $17.6(35 / 199)$ & $17.6(36 / 205)$ & $21.2(47 / 222)$ \\
\hline CR-S. marcescens & $\mathrm{I} .5(\mathrm{I} / 65)$ & I.5 (I/65) & II.4 (8/70) & $4.5(3 / 66)$ & $12.2(6 / 49)$ \\
\hline ESBL-E. coli & $61.6(106 / 172)$ & $64.3(117 / 182)$ & $57.6(1 / 4 / 198)$ & $62.8(137 / 218)$ & $50.4(127 / 252)$ \\
\hline ESBL-K. pneumoniae & $41.2(70 / 170)$ & $32.1(60 / 187)$ & 24.6 (49/199) & $28.3(58 / 205)$ & $18(40 / 222)$ \\
\hline ESBL-P. mirabilis & $33.8(22 / 65)$ & $31.8(21 / 66)$ & $21.4(15 / 70)$ & $20.5(16 / 78)$ & $1.9(1 / 54)$ \\
\hline CR-A. baumannii & $64.4(116 / 180)$ & $64(110 / 172)$ & $70.8(148 / 209)$ & $80.2(162 / 202)$ & $69.3(113 / 163)$ \\
\hline CR-P. aeruginosa & $29.2(54 / 185)$ & $31.5(56 / 178)$ & $30.8(62 / 201)$ & 34.1 (70/205) & $29.4(45 / 153)$ \\
\hline
\end{tabular}

Notes: Carbapenem-resistant isolates are defined as Enterobacterales, which were resistant to any of the resistant to meropenem, imipenem, and ertapenem; A. baumannii and $P$. aeruginosa which as any of the resistant to meropenem and imipenem.

Abbreviations: $C R$, carbapenem resistant; ESBL, extended-spectrum $\beta$-lactamases. 
Table 3 Prevalence of Multidrug-Resistant Gram-Negative Isolates in Different Infection Types

\begin{tabular}{|c|c|c|c|c|}
\hline \multirow[t]{2}{*}{ Organism } & \multicolumn{4}{|c|}{ \% (No. of Isolates) } \\
\hline & BSIs & IAls & RIs & UTIs \\
\hline CR-A. baumannii & $73.2(298 / 407)$ & $72.4(84 / 116)$ & $72.9(145 / 199)$ & $56.4(22 / 39)$ \\
\hline CR-C. freundii & $7(3 / 43)$ & $4.9(4 / 82)$ & $6.9(7 / 101)$ & $8.9(11 / 124)$ \\
\hline CR-K. aerogenes & $1.8(1 / 56)$ & $8(4 / 50)$ & $3.5(4 / 115)$ & $2.3(1 / 44)$ \\
\hline CR-E. cloacae & $7.4(18 / 244)$ & $8.4(10 / 119)$ & $7.4(20 / 270)$ & $14.4(13 / 90)$ \\
\hline CR-E. coli & $2.4(|7 / 7| 0)$ & $1.5(2 / 134)$ & 9.1 (2/22) & $\mathrm{I}(1 / 100)$ \\
\hline CR-K. pneumoniae & $14.2(80 / 564)$ & $14.5(19 / 131)$ & $14.4(15 / 104)$ & $25.4(|8 / 7|)$ \\
\hline CR-P. aeruginosa & $47(156 / 332)$ & $44(66 / 150)$ & $46.8(102 / 218)$ & $44.3(35 / 79)$ \\
\hline CR-S. marcescens & $12.3(10 / 8 \mid)$ & $6.9(2 / 29)$ & $5.7(7 / / 22)$ & $0(0 / 38)$ \\
\hline ESBL-E. coli & $57.9(4 \mid I / 7 / 0)$ & $54.5(73 / 134)$ & $59.1(13 / 22)$ & $63(63 / 100)$ \\
\hline ESBL-K. pneumoniae & $27.7(156 / 564)$ & $26.7(35 /|3|)$ & $29.8(31 / 104)$ & $38(27 / 7 I)$ \\
\hline ESBL-P. mirabilis & $10.9(5 / 46)$ & $16.7(6 / 36)$ & $16.1(10 / 62)$ & $28.9(39 / 135)$ \\
\hline
\end{tabular}

Notes: Carbapenem-resistant isolates are defined as Enterobacterales, which were resistant to any of the resistant to meropenem, imipenem, and ertapenem; A. baumannii and $P$. aeruginosa which as any of the resistant to meropenem and imipenem.

Abbreviations: BSIs, bloodstream infections; IAls, intra-abdominal infections; Rls, respiratory infections; UTls, urinary tract infections; CR, carbapenem resistant; ESBL, extended-spectrum beta-lactamases.

\section{Cumulative MIC Analysis of E. coli, K. pneumoniae, P. aeruginosa, and}

\section{A. baumannii Against Antimicrobial Agents}

Figure 1 shows the cumulative distribution of the MICs of different types of antimicrobial agents against the four majors gram-negative bacteria (E. coli, $K$. pneumoniae, $P$. aeruginosa, and A. baumannii). All three carbapenems had an MIC of less than $1 \mathrm{mg} / \mathrm{L}$ for more than $95 \%$ of the E. coli isolates (Figure 1A). When the MIC value was between $0.25 \mathrm{mg} / \mathrm{L}$ and $8 \mathrm{mg} / \mathrm{L}$, the difference between the curve of $K$. pneumoniae and the curve of E. coli was clear. For the $P$. aeruginosa curve (Figure 1B), the MIC of meropenem was lower than $2 \mathrm{mg} / \mathrm{L}$ for $70 \%$ of the isolates, and the MIC of imipenem was lower than $2 \mathrm{mg} / \mathrm{L}$ for $60 \%$ of the isolates. The MIC of meropenem and imipenem was greater than $8 \mathrm{mg} / \mathrm{L}$ in more than $75 \%$ of the isolates of A. baumannii. Figure $1 \mathrm{C}$ and D show the cumulative MIC percentage curves of the three generation cephalosporins against the four majors gram-negative bacteria. For $K$. pneumoniae, when the MIC was below $0.5 \mathrm{mg} / \mathrm{L}$, the proportion of ceftriaxone- and cefotaxime-susceptible isolates was higher than the proportion of ceftazidime-susceptible isolates. When the MIC was above $0.5 \mathrm{mg} / \mathrm{L}$, the proportion of ceftriaxone- and cefotaxime-susceptible isolates was lower than that of ceftazidime-sensitive isolates. This phenomenon also appeared in the curve of E. coli, but the demarcated MIC value became $0.25 \mathrm{mg} / \mathrm{L}$. Ceftazidime has significantly higher antibacterial activity against P. aeruginosa than against $A$. baumannii. For ciprofloxacin and levofloxacin, an MIC value lower than $4 \mathrm{mg} / \mathrm{L}$ was effective for more than $70 \%$ of the isolates of K. pneumoniae, and an MIC value lower than $1 \mathrm{mg} / \mathrm{L}$ was noted for $60-70 \%$ of the isolates. For ciprofloxacin and levofloxacin (Figure 1E), an MIC value below $2 \mathrm{mg} / \mathrm{L}$ was feasible for more than $40 \%$ of the isolates of $E$. coli, and an MIC value below $0.5 \mathrm{mg} / \mathrm{L}$ was effective for $35-40 \%$ of the isolates. For A. baumannii (Figure 1F), when the treatment concentrations of ciprofloxacin and levofloxacin were below $2 \mathrm{mg} / \mathrm{L}$, the MIC distribution of the two drugs was not appreciably different. When the concentration was above $2 \mathrm{mg} / \mathrm{L}$, more than $70 \%$ of the isolates were responsive to less than $8 \mathrm{mg} / \mathrm{L}$ of levofloxacin, and only about $30 \%$ of the isolates responded to less than $8 \mathrm{mg} / \mathrm{L}$ of ciprofloxacin. The differences in the distribution of the MIC values of levofloxacin and ciprofloxacin against $P$. aeruginosa were mainly concentrated between the MIC values of $0.125 \mathrm{~m} / \mathrm{L}$ and $1 \mathrm{mg} / \mathrm{L}$. The MIC distribution of cefoperazone-sulbactam and piperacillin-tazobactam on $E$. coli is shown in Figure $1 \mathrm{G}$. When the MIC value was above $16 \mathrm{mg} / \mathrm{L}$, the MIC distributions of the two agents to E. coli was not much different. The cumulative MIC curves of cefoperazonesulbactam and piperacillin-tazobactam on $P$. aeruginosa almost coincided (Figure 1H). For A. baumannii, when the MIC value was above $16 \mathrm{mg} / \mathrm{L}$, cefoperazone-sulbactam had better antibacterial activity in vitro.

\section{Prevalence of Major Carbapenemase Genes in All CRE Isolates}

Of the 295 CRE isolates, 233 were tested by PCR for the major carbapenemase genes. As shown in Table 4, in total, 

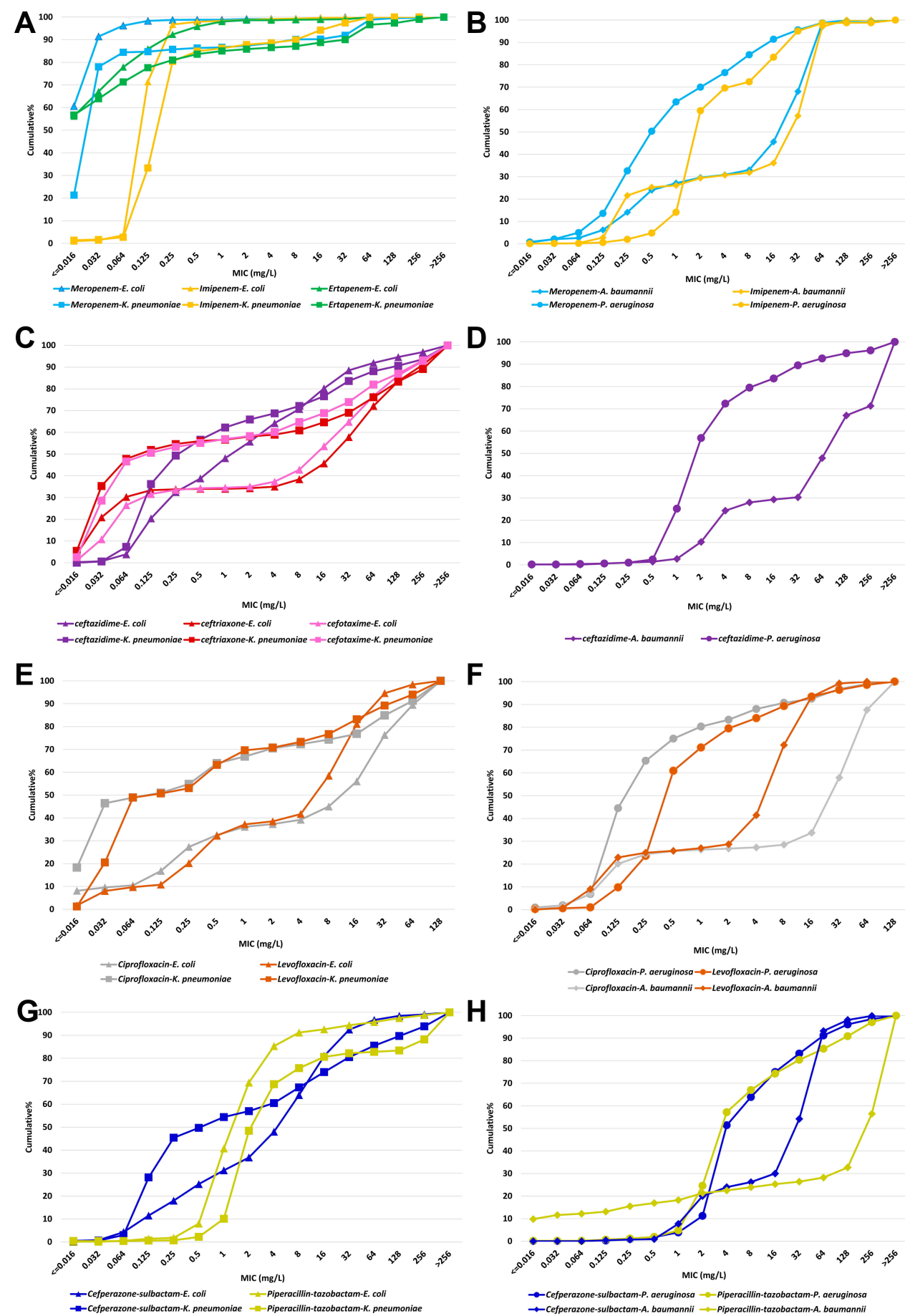

Figure I Cumulative MIC of E. coli, (K) pneumoniae, (P) aeruginosa, and A. baumannii against antimicrobial agents. (A and B) Cumulative MIC of E. coli, K. pneumoniae, $P$. aeruginosa, and A. baumannii against carbapenems. (C and D) Cumulative MIC of $E$. coli, $K$. pneumoniae, P. aeruginosa, and A. baumannii against major cephalosporins including ceftazidime, ceftriaxone, and cefotaxime. (E and F) Cumulative MIC of E. coli, K. pneumoniae, P. aeruginosa, and A. baumannii against quinolones. (G and $\mathbf{H})$ Cumulative MIC of E. coli, K. pneumoniae, P. aeruginosa, and A. baumannii against cefoperazone-sulbactam and piperacillin-tazobactam.

129 K. pneumoniae were tested by PCR: 92 isolates were detected to carry the $b l a_{\mathrm{KPC}-2}$ gene, 14 isolates carried the $b l a_{\mathrm{NDM}}$ gene, and one isolate carried the bla $a_{\mathrm{IMP}}$ gene. Forty-four E. cloacae were tested for carbapenemase genes by PCR; of these, 2 carried the $b l a_{\mathrm{KPC}}$ gene,
7 carried the $b l a_{\mathrm{NDM}}$ gene, and 2 carried the $b l a_{\mathrm{IMP}}$ gene; 33 isolates did not carry these three carbapenemase genes. Twenty-five isolates of $C$. freundii were tested by PCR; of these, 9 isolates carried $b l a_{\mathrm{NDM}}, 2$ isolates carried $b l a_{\mathrm{KPC}}$, and 2 isolates carried both the $b l a_{\mathrm{KPC}}$ and $b l a_{\mathrm{IMP}}$ genes. 
Table 4 Prevalence of Major Carbapenemase Genes in All Carbapenem-Resistant Enterobacterales Isolates

\begin{tabular}{|c|c|c|c|c|c|c|c|c|c|c|}
\hline \multirow[t]{2}{*}{ Organisms } & \multicolumn{7}{|c|}{ Carbapenemase Gene } & \multirow{2}{*}{$\begin{array}{l}\text { PCR } \\
\text { Negative }\end{array}$} & \multirow{2}{*}{$\begin{array}{l}\text { Not } \\
\text { Tested }\end{array}$} & \multirow[t]{2}{*}{ Total } \\
\hline & $b / a_{K P C-2}$ & $b l a_{\mathrm{KPC}-2+1 \mathrm{MP}-1}$ & $b l a_{\text {NDM-I }}$ & $b l a_{\text {NDM-5 }}$ & $b^{\prime} a_{\text {NDM-7 }}$ & $b / a_{1 M P-1}$ & bla $a_{\text {IMP-4 }}$ & & & \\
\hline K. pneumoniae & 92 & & 10 & 2 & 2 & & I & 22 & 20 & 149 \\
\hline E. cloacae & 2 & & 5 & 2 & & 1 & 1 & 33 & 24 & 68 \\
\hline C. freundii & 2 & 2 & 9 & & & & 1 & II & 2 & 27 \\
\hline E. coli & 1 & & 2 & 4 & & & & 9 & 6 & 22 \\
\hline S. marcescens & 3 & & & & & & & $\mathrm{II}$ & 5 & 19 \\
\hline K. aerogenes & 1 & & & & & & & 4 & 5 & 10 \\
\hline Total & 101 & 2 & 26 & 8 & 2 & I & 3 & 90 & 62 & 295 \\
\hline
\end{tabular}

Twenty-five isolates of $E$. coli were tested by PCR; of these, six isolates carried $b l a_{\mathrm{NDM}}$, and one isolate carried $b l a_{\mathrm{KPC}}$.

\section{Discussion}

In comparison with the last CMSS surveillance report, the resistance rates of $K$. pneumoniae and $A$. baumannii to carbapenem drugs are gradually increasing. Thus, the current status of antimicrobial resistance is grim. We found that the incidence of major multidrug-resistant bacteria is different in different types of infection. The prevalence of ESBL-E. coli, ESBL-K. pneumoniae, ESBL-P. mirabilis, and CRKP from UTIs were significantly higher than those from the other three infection types (BSIs, IAIs, and RIs).

Our data is based on the standard agar dilution method and micro-broth dilution method, which can provide accurate MIC data for clinical use. For anti-infective treatment, MIC data is essential in the treatment of severe infections. When providing individualized treatment, it is necessary to combine pharmacokinetic/pharmacodynamic (PK/PD) data and MIC determinations to calculate the dosage of the antimicrobial agent(s) to be administered to the patient. ${ }^{18}$ The clinical application of PK/PD theory is one of the many reliable strategies that are effective in realizing the therapeutic potential of existing antimicrobial agents. ${ }^{19}$ The CLSI had lowered the susceptible breakpoint of quinolone for the treatment of Enterobacterales and P. aeruginosa in the 2019 update. ${ }^{14}$ Especially in the treatment of severely infected patients, new breakpoints are used to determine the dosage of antimicrobial agents, and a corresponding area under the curve (AUC)/MIC target can be achieved by evaluating the corresponding drug dosage. ${ }^{20-22}$ From the cumulative MIC of E. coli, $K$. pneumoniae, and $P$. aeruginosa against levofloxacin and ciprofloxacin, we can see that the new breakpoint has a lower effect on the susceptibility rate of the two drugs in vitro.

Our multi-center research shows that the prevalence of CRKP in China has been increasing over the past ten years. The prevalence of CRKP increased from 7.6\% in 2010 to $21.2 \%$ in 2018 . This result is consistent with the surveillance conducted by China's most significant drug surveillance network: China antimicrobial resistance surveillance system (CARSS). ${ }^{23}$ The mortality after CRKP infection is very high. In some studies, the mortality rate of CRKP bacteremia was as high as $50-70 \% .^{24,25}$ In this study, the primary resistance mechanism of CRKP was caused by carbapenemase encoded by plasmid-mediated $b l a_{\mathrm{KPC}}$, which is consistent with our previous CRE-related studies. ${ }^{26-28}$ Several studies have shown that $\mathrm{CRKP}$ carrying bla $_{\mathrm{KPC}}$-type plasmids can occur in large-scale outbreaks or spread in hospitals. These reports have highlighted the considerable obstacles clinicians face in the prevention and control of nosocomial infections. ${ }^{29,30}$ At present, in the clinical treatment of CRKP infection, it is recommended to provide a combination of drugs that are sensitive to in vitro antimicrobial susceptibility tests within the allowable range, extend the infusion time, and increase the dose to achieve the goal of T\%> MIC. ${ }^{19,31}$ Our data show that tigecycline, colistin, and amikacin also maintain high in vitro activity against $K$. pneumoniae. Meropenem has shown in vitro activity against other Enterobacterales, including $E$. coli, E. cloacae, and $C$. freundii.

The susceptibility of $A$. baumannii to carbapenems declined significantly from 2010 to 2018 . In the treatment of CRAB, there are fewer options for antimicrobial agents as indicated by the in vitro susceptibility tests; thus, the treatment of a CRAB infection often requires combined treatment. Attention should be paid to the MIC of antimicrobial agents as well. ${ }^{32}$ The susceptibility of $P$. aeruginosa to carbapenem is increasing, and the 
susceptibility to other anti-pseudomonas drugs is also increasing. Simultaneously, domestic CHINET research shows that the incidence of carbapenem-resisitant $P$. aeruginosa was also decreasing, in accordance with the results of this study. ${ }^{33}$

\section{Conclusion}

The data of the CMSS from 2010 to 2018 show that the current situation of antimicrobial resistance in China is severe. The results indicate an increase in $K$. pneumoniae resistance to carbapenems over time, mainly owing to KPC-type carbapenemase production. A. baumannii was severely resistant to carbapenems in China. Ongoing MICbased resistance surveillance, like CMSS, provides additional data for clinical anti-infective treatment.

\section{Abbreviations}

CARSS, China antimicrobial resistance surveillance system; CLSI, Clinical and Laboratory Standards Institute; CMSS, Chinese Meropenem Surveillance Study; CRAB, carbapenem-resistant Acinetobacter baumannii; CRE, carbapenemresistant Enterobacterales; ESBL, extended-spectrum beta-lactamase; MICs, minimum inhibitory concentrations; MYSTIC, Meropenem Yearly Susceptibility Test Information Collection; BSIs, bloodstream infections; IAIs, intra-abdominal infections; RIs, respiratory infections; UTIs, urinary tract infections.

\section{Funding}

The CMSS project was supported by a research grant from Sumitomo Pharmaceuticals (Suzhou) Co., Ltd.

\section{Disclosure}

The authors report no conflicts of interest in this work.

\section{References}

1. Tacconelli E, Cataldo MA, Dancer SJ, et al. ESCMID guidelines for the management of the infection control measures to reduce transmission of multidrug-resistant Gram-negative bacteria in hospitalized patients. Clin Microbiol Infect. 2014;20(Suppl 1):1-55. doi:10.1111/1469-0691.12427

2. Wright H, Bonomo RA, Paterson DL. New agents for the treatment of infections with Gram-negative bacteria: restoring the miracle or false dawn? Clin Microbiol Infect. 2017;23:704-712. doi:10.1016/j. cmi.2017.09.001

3. Horcajada JP, Montero M, Oliver A, et al. Epidemiology and treatment of multidrug-resistant and extensively drug-resistant pseudomonas aeruginosa infections. Clin Microbiol Rev. 2019;32:e0031-19.

4. Morency-Potvin P, Schwartz DN, Weinstein RA. Antimicrobial stewardship: how the microbiology laboratory can right the ship. Clin Microbiol Rev. 2017;30:381-407. doi:10.1128/CMR.00066-16
5. Wong D, Nielsen TB, Bonomo RA, et al. Clinical and pathophysiological overview of acinetobacter infections: a century of challenges. Clin Microbiol Rev. 2017;30:409-447.

6. CDC. Antibiotic Resistance Threats in the United States, 2019. Atlanta, GA: U.S. Department of Health and Human Services, CDC; 2019.

7. David S, Reuter S, Harris SR, et al. Epidemic of carbapenem-resistant Klebsiella pneumoniae in Europe is driven by nosocomial spread. Nat Microbiol. 2019;4:1919-1929. doi:10.1038/ s41564-019-0492-8

8. Brolund A, Lagerqvist N, Byfors S, et al. Worsening epidemiological situation of carbapenemase-producing Enterobacteriaceae in Europe, assessment by national experts from 37 countries, July 2018. Euro Surveill. 2019;24:1900123. doi:10.2807/1560-7917.ES.2019.24.9.19 00123

9. Jones RN, Mendes C, Turner PJ, et al. An overview of the meropenem yearly susceptibility test information collection (MYSTIC) program: 1997-2004. Diagn Microbiol Infect Dis. 2005;53:247-256. doi:10.1016/j.diagmicrobio.2005.10.006

10. Masterton RG, Kuti JL, Turner PJ, et al. The OPTAMA programme: utilizing MYSTIC (2002) to predict critical pharmacodynamic target attainment against nosocomial pathogens in Europe. J Antimicrob Chemother. 2005;55:71-77. doi:10.1093/jac/dkh511

11. Korten V, Ulusoy S, Zarakolu P, et al. Antibiotic resistance surveillance over a 4-year period (2000-2003) in Turkey: results of the MYSTIC program. Diagn Microbiol Infect Dis. 2007;59:453-457. doi:10.1016/j.diagmicrobio.2007.06.016

12. Rhomberg PR, Deshpande LM, Kirby JT, et al. Activity of meropenem as serine carbapenemases evolve in US Medical Centers: monitoring report from the MYSTIC program (2006). Diagn Microbiol Infect Dis. 2007;59:425-432. doi:10.1016/j.diagmicrobio.2007.05.009

13. Wang $\mathrm{H}$, Chen $\mathrm{M}$, Ni $\mathrm{Y}$, et al. Antimicrobial resistance among clinical isolates from the Chinese Meropenem Surveillance Study (CMSS), 2003-2008. Int J Antimicrob Agents. 2010;35:227-234. doi:10.1016/j.ijantimicag.2009.11.010

14. CLSI. Performance Standards for Antimicrobial Susceptibility Testing. 29th ed. CLSI supplement M100. Wayne, PA: Clinical and Laboratory Standards Institute; 2019.

15. The European Committee on Antimicrobial Susceptibility Testing. Breakpoint tables for interpretation of MICs and zone diameters. Version 10.0. 2020. Available from: http://www.eucast.org.

16. Yang Q, Wang H, Sun H, et al. Phenotypic and genotypic characterization of Enterobacteriaceae with decreased susceptibility to carbapenems: results from large hospital-based surveillance studies in China. Antimicrob Agents Chemother. 2010;54:573-577. doi:10.11 28/AAC.01099-09

17. Wang $\mathrm{X}, \mathrm{Xu} \mathrm{X}, \mathrm{Li} \mathrm{Z}$, et al. An outbreak of a nosocomial NDM-1-producing Klebsiella pneumoniae ST147 at a teaching hospital in mainland China. Microb Drug Resist. 2014;20:144-149. doi: $10.1089 / \mathrm{mdr} .2013 .0100$

18. Delattre IK, Taccone FS, Jacobs F, et al. Optimizing $\beta$-lactams treatment in critically-ill patients using pharmacokinetics/pharmacodynamics targets: are first conventional doses effective? Expert Rev Anti Infect Ther. 2017;15:677-688. doi:10.1080/14787210.2017.1338139

19. Rodriguez-Bano J, Gutierrez-Gutierrez B, Machuca I, et al. Treatment of infections caused by extended-spectrum-beta-lactamase-, AmpC-, and carbapenemase-producing Enterobacteriaceae. Clin Microbiol Rev. $2018 ; 31$.

20. Bulik CC, Bader JC, Zhang L, et al. PK-PD compass: bringing infectious diseases pharmacometrics to the patient's bedside. $J$ Pharmacokinet Pharmacodyn. 2017;44:161-177. doi:10.1007/s10928-017-9518-0

21. Drusano GL, Preston SL, Fowler C, et al. Relationship between fluoroquinolone area under the curve: minimum inhibitory concentration ratio and the probability of eradication of the infecting pathogen, in patients with nosocomial pneumonia. $J$ Infect Dis. 2004;189:1590-1597. doi: $10.1086 / 383320$ 
22. Preston SL, Drusano GL, Berman AL, et al. Levofloxacin population pharmacokinetics and creation of a demographic model for prediction of individual drug clearance in patients with serious community-acquired infection. Antimicrob Agents Chemother. 1998;42:1098-1104. doi:10.1128/AAC.42.5.1098

23. China Antimicrobial Resistance Surveillance System. China antimicrobial resistance surveillance report, 2018. Available from: http:// carss.cn/Report/Details/648. Accessed July 23, 2020.

24. Wang X, Wang Q, Cao B, et al. Impact of combination therapy vs monotherapy on mortality from carbapenem-resistan Enterobacteriaceae bacteremia: a retrospective observational study from a Chinese network. Antimicrob Agents Chemother. 2018. doi:10.1128/AAC.01511-18

25. Tzouvelekis LS, Markogiannakis A, Psichogiou M, et al. Carbapenemases in Klebsiella pneumoniae and other Enterobacteriaceae: an evolving crisis of global dimensions. Clin Microbiol Rev. 2012;25:682-707. doi:10.1128/CMR.05035-11

26. Zhang Y, Wang Q, Yin Y, et al. Epidemiology of carbapenem-resistant Enterobacteriaceae infections: report from the China CRE Network. Antimicrob Agents Chemother. 2018;62.

27. Wang Q, Wang X, Wang J, et al. Phenotypic and genotypic characterization of carbapenem-resistant Enterobacteriaceae: data from a longitudinal large-scale CRE study in China (2012-2016). Clin Infect Dis. 2018;67:S196-S205. doi:10.1093/cid/ciy660
28. Li H, Zhang J, Liu Y, et al. Molecular characteristics of carbapenemase-producing Enterobacteriaceae in China from 2008 to 2011: predominance of KPC-2 enzyme. Diagn Microbiol Infect Dis. 2014;78:63-65. doi:10.1016/j.diagmicrobio.2013.10.002

29. Ruppe E, Olearo F, Pires D, et al. Clonal or not clonal? Investigating hospital outbreaks of KPC-producing Klebsiella pneumoniae with whole-genome sequencing. Clin Microbiol Infect. 2017;23 (7):470-475. doi:10.1016/j.cmi.2017.01.015

30. Snitkin ES, Zelazny AM, Thomas PJ, et al. Tracking a hospital outbreak of carbapenem-resistant Klebsiella pneumoniae with whole-genome sequencing. Sci Transl Med. 2012;4:148ra16-ra16. doi:10.1126/scitranslmed.3004129

31. Falagas ME, Tansarli GS, Ikawa K, et al. Clinical outcomes with extended or continuous versus short-term intravenous infusion of carbapenems and piperacillin/tazobactam: a systematic review and meta-analysis. Clin Infect Dis. 2013;56:272-282. doi:10.1093/cid/cis857

32. Guan X, He L, Hu B, et al. Laboratory diagnosis, clinical management and infection control of the infections caused by extensively drug-resistant Gram-negative bacilli: a Chinese consensus statement. Clin Microbiol Infect. 2016;22(Suppl 1):S15-25. doi:10.1016/j.cmi.2015.11.004

33. Hu FP, Guo Y, Zhu DM, et al. Resistance trends among clinical isolates in China reported from CHINET surveillance of bacterial resistance, 2005-2014. Clin Microbiol Infect. 2016;22:S9-14. doi:10.1016/j.cmi.2016.01.001
Infection and Drug Resistance

\section{Publish your work in this journal}

Infection and Drug Resistance is an international, peer-reviewed openaccess journal that focuses on the optimal treatment of infection (bacterial, fungal and viral) and the development and institution of preventive strategies to minimize the development and spread of resistance. The journal is specifically concerned with the epidemiology of
Dovepress

antibiotic resistance and the mechanisms of resistance development and diffusion in both hospitals and the community. The manuscript management system is completely online and includes a very quick and fair peerreview system, which is all easy to use. Visit http://www.dovepress.com/ testimonials.php to read real quotes from published authors. 